ren, querstehenden, hornartigen Zahnblättchen. Denn wie soll ersterer, selbst wenn er mit grösster Behutsamkeit zu Werke geht, das zarte Junge so leise anfassen, dass er es nicht mit den Zähnen verletzt? zumal wenn er nicht etwa den Leib, sondern den Hals desselben in den Schnabel nimmt? Ja, wenn das Junge sich dabei etwa gan $\iota$ regungslos verhielte! Das wird es jedoch schwerlich thun. Folglich darf es die Alte nicht gar zu leise anfassen, weil es ihr sonst durch sein Zappeln entgleiten würde.

Ich bin daher der Meinung, dass alle Beobachtungen, die von einem Forttragen der Jungen im Schnabel sprechen, auf einem Fehler im Sehen beruhen, der sich aus den Umständen leicht erklären lässt. Nämlich, ich glaube: es findet überall dasjenige Verfahren Statt, welches einige gute forstmännische Beobachter bei der W aldschnepfe wahrgenommen haben.

Dieses bestand aber darin, dass die Alte den Hals des Jungen $\mathrm{zw}$ ischen ihren zurückgebogenen eigenen $\mathrm{Hals}$ und die Brust einklemmte. Also: Weich auf Weich. Damit fällt alles Quetschen, Stechen, Kratzen und sonstiges Verletzen von selbst weg. Indem nun die Mutter so mit ihrem Halse gleichsam eine Schlinge bildet, muss sie, um den Hals des Kleinen in derselben festzuhalten, ihren Kopf und Schnabel weit nach vorn und nach unten zurücklegen. So ragt denn auf der einen Seite der Kopf des Jungen, auf der anderen Seite der Leib desselben, unter ihrem Schnabel hervor; und hierdurch entsteht bereits auf geringe Entfernung hin der Anschein, als habe sie das Kleine im Schnabel. Nur ganz in der Nähe kann ein genauer Beobachter das Richtige wahrnehmen, (wie diess eben bei der Waldschnepfe gelungen ist.) Daher die vielfachen Täuschungen über das angebliche Fort- oder Herabtragen der Jungen "im Schnabel!“

Fände übrigens das Letztere Statt, so würde und müsste die Alte dabei mit ausgestrecktem Halse fliegen. Es genügt also, darauf zu achten, dass sie das nicht thut.

Berlin, den 29. Januar 1860.

Gloger.

\title{
Allgemeine Bemerkungen und über einige Vögel Belgiens.
}

$$
\text { Von }
$$

Ch. F. Dubois.

In der ersten Hälfte dieses Jahrhunderts wurde durch fleissiges Beobachten und durch Anlegen bedeutender ornithologischer Sammlungen, 
diese schöne Wissenschaft um ein Grosses erweitert, ebenso aber auch mit manchen neuen Arten vermehrt, denen leider jetzt, in der zweiten Hälfte, das traurige Schicksal zufällt, zum Theil wieder zu Grabe getragen zu werden. - Ich muss gestehen, dass man viel zu schnell damit verfährt, manche solcher zweifelhaften Vögel aus der Reihe der Arten der Ornithologie Europas zu verdrängen, und obschon man einstweilen nur hiermit den Anfang gemacht hat, steht zu erwarten, dass die exotischen Vögel dieselbe Revue erleiden werden. Freilich bieten manche Arten zu unhaltbare Kennzeichen dar, um sie als gute Art anerkennen zu können, wodurch ihr sicheres Loos ist, dass sie bald in Vergessenheit gerathen, und die unausbleibliche Folge, dass sie gewöhnlich von selbst wieder verschwinden.

Nicht so leicht dürfte es mit den, in unsrer Zeit in grosser Menge entstandenen neuen Gattungen sein! Es ist nicht mehr weit davon, dass jede Art auch ihre eigene Gattung bildet und scheint es eine wahre Krankheit unsrer Zeit zu sein, alles durch einander zu werfen, damit später unsre Nachfolger wieder neue Arbeit finden, dieses undurchdringliche Labyrinth von Gattungen und Arten in ihre natürlichen Schranken zurückzubringen; denn es sind Gattungen entstanden, ohne dass jemals eine Aufklärung über ihre Kennzeichen gegeben wurde. Diess ist zwar nicht zu verwundern, wenn man in Betracht zieht, dass keine zu geben waren, oder, man müsste denn dergleichen geringfügige Kennzeichen angeben, welche nur wirkliche Artkennzeichen sind, oder zu dem Mittel greifen, die Farbe des Gefieders dazu zu benutzen. Dieses traurige Schicksal hat aber nicht allein die Ornithologie erleiden müssen, sondern es hat auch durch das ganze Naturreich um sich gegriffen, und diess nennt man ein riesenhaftes Fortschreiten der Wissenschaft! - Es war zwar vorauszusehen, dass bedeutende Aenderungen und Verbesserungen nach Linné's Zeit entstehen mussten; allein, nachdem einmal diese Arbeit begonnen, glaubte ein Jeder Theil daran nehmen zu müssen, mitunter Männer ganz ohne alle ornithologische Kenntnisse. Es handelt sich nicht mehr um die wirkliche Wissenschaft, sondern um den Namen ein System oder ein derartiges Verzeichniss von Vögeln eines Landstriches von Europa geschrieben zu haben. Weil nun aber in einem solchen Verzeichnisse keine neue Vögel gegeben werden können, so müssen denn neue Systeme und Gattungen aufgestellt werden, und um diesen Zweck leichter zu erreichen, nimmt man eines der alten Werke von Aldrovand, Gessner, Charlet, Klein u. s. w. zur Hand, benutzt deren lateinische Namen, und giebt solche, ohne diese Männer nur im geringsten zu erwähnen. - Unterwirft man nun solche Arbeiten, welche nur das 
Resultat eines Studiums am Schreibtische sind, einer genaueren Prüfung, so ergibt sich, dass sie wenig oder gar keinen Werth haben und dabei meistens einen deutlichen Beweis von Unerfahrenheit in der Natur zeigen. - Wer sich daher dem Studium der Natur unterziehen will, der begebe sich hinaus in Gottes freie Natur, unter den blau gewölbten Himmel, auf Felder und in Wälder, wo mit jedem Schritte und je weiter man in dieses Heiligthum dringt, je deutlicher die Grösse unsers gütigen Schöpfers zu erkennen ist, wo alsdann dem Naturforscher die reinsten und heiligsten Gefühle das Herz durchdringen und nicht selten Thränen der Liebe und des Dankes hervorpressen! Wie wohlthuend ist es nicht dem Menschen, selbst dem Ungebildetsten, in den schönen Morgenstunden, wenn die ersten Strahlen der Sonne auftauchen und die Lerche sich hoch in die Lüfte erhebt und ihren angenehmen Gesang ertönen lässt; wie viele andere Stimmen begrüssen nicht den beginnenden Tag; die Pflanzen, noch vom nächtlichen Taue mit Krystall-Tropfen befeuchtet, richten verjüngt ihre Häupter der Sonne zu, um langsam ihre Blumen zu öfnen; Alles athmet neues Leben; die Insecten kommen aus ihren Verstecken hervor, die Schmetterlinge flattern von Blume zu Blume; die Raupe kriecht an den Pflanzen umher, um ihre grosse Gefrässigkeit zu befriedigen. Ja überall giebt es der Beobachtungen in Menge, und bei näherer Untersuchung, wie Manches auch über europäische Vögel. Wem die Gelegenheit geboten ist das Leben und Treiben der Vögel, deren Fortpflanzung, die Erziehung ihrer Jungen zu belauschen, wer sich diesem mit freudiger Liebe und Ausdauer unterziehen will, wird sicherlich noch Vieles zu deren Naturgeschichte beitragen können, wie uns bereits so manche werthvolle Mittheilungen in diesem Journal bewiesen haben. Hierdurch wird die Ornithologie bereichert, obschon mancher dies für Nebensache halten mag.

Einen Beweis, wie unsicher oft die neuen Genera sind, liefert z. B. die Fringilla incerta, welche Bonaparte als Carpodacus incertus aufführt, nachher von dieser, ihr wirklich zugehörenden Gattung nimmt, und in sein Genus Chlorospiza stellt, während der Vogel doch nur eine Abänderung von Carpodacus erythrinus ist, wie man auch in diesem Journal, Jahrg. 1856, Seite 313 angegeben findet. Derartige Irrthümer finden sich öfters.

Unter den bezweifelten Arten dürfte wohl mit einigem Recht die Pyrrhula coccinea Selys ihren Platz standhaft unter den Vögeln Europas behaupten, da sie einen bedeutenden Grössen-Unterschied gegen Pyrrhula vulgaris zeigt, wie man sich auch leicht an frischen oder lebenden Exemplaren überzeugen kann. Man hat zu oft Gelegenheit diese Vögel 
im Fleische zu erhalten, welche bei den hiesigen Vogelhändlern als , grand Bouvereuil" von der Pyrrhula vulgaris unterschieden werden. Pyrrhula coccinea besuchte Belgien in verschiedenen Jahren, oft in mehreren Jahren gar nicht, dann aber wieder meistens zu grossen Flügen vereinigt, wie man es bei Pyrrhula vulgaris hier niemals bemerkt. Letztere nistet auch hier, hingegen Pyrrhula coccinea besucht uns nur für die Winterszeit, wo sie mitunter in so grossen Mengen gefangen wurden, dass sie zum Verspeisen Dutzendweise bei den Wildprethändlern zum Verkaufe auslagen.

Ebenso wird die Verschiedenheit der Carduelis Holboelli, canescens und linaria bezweifelt, obgleich bei diesen Arten auf die Grösse der Schnäbel nicht allein zu achten ist, da solche im Allgemeinen nicht sehr standhaft in ihrer Stärke oder Grösse bleiben; unmöglich aber kann $C$. Holboelli und $C$. canescens ein und derselben Art zugehören. $C$. Holboelli ist vom Körper reichlich um ein Drittel grösser und hat einen viel stärkeren Schnabel, welcher sehr dem Schnabel von C. elegans ähnlich ist, auch sind dessen Flügel bedeutend grösser und haben eine mehr zugespitzte Form. Dieser Vogel erscheint sehr selten in Belgien; sollte derselbe nur, wie man behaupten will, ein recht grosses Exemplar der C. linaria sein, so würden solche nur zufällig und einzeln vorkommen, aber nicht zu Paaren, oder gar mehrere Stücke, wie solche ¡m Jahre 1856 hier gefangen wurden. C. canescens ist die kleinste von allen dreien und besitzt den kleinsten Schnabel, das Gefieder zeigt eine mehr rostbraune Färbung. Sie erscheinen hier alle Jahre gegen Ende September, oft in so grosser Menge, dass mehrere Hunderte auf den hiesigen Markt gebracht und unter dem Namen "petit cabaret" verkauft werden. Darunter befinden sich auch gewöhnlich Junge ohne rothe Scheitelplatte. C. linaria erscheint hingegen viel später und in weit geringerer Anzahl, in manchen Jahren gar nicht, auch ist ihr Gefieder nicht von so rostbrauner Farbe wie das der C.canescens, sondern mehr graubraun.

Herr de Selys - Longchamps sagt in der Naumannia, Jahrg. 1856, Seite 392, er zweifele an der Verschiedenheit des Turdus fuscatus und Turdus Naumannii; derselbe hat aber erstere Art, welche vor mehreren Jahren hier gefangen wurde, iu der Académie Royale des sciences de Bruxelles als Turdus Naumannii beschrieben. Als ich im Jahre 1853 denselben Vogel für meine Abbildungen der „Vögel Belgiens" benutzte, bemerkte ich dessen Irrthum, da das Exemplar ein junger Vogel von $C$. fuscatus war. Das einzige Exemplar des $\boldsymbol{T}$. Naumannii, welches hier in Belgien gefangen wurde, habe ich selbst 
im Fleische erhalten. Diese Drossel hat dieselbe Gestalt wie T.iliacus, und alle Obertheile nebst Flügel sind mehr olivenfarbig braun, dagegen ist $T$. fuscatus etwas grösser und hat ganz die gedrungene Gestalt des T. pilaris; die Obertheile und Flügel sind mehr rothbraun, selbst schon beim jungen Vogel. Prinz Bonaparte scheint eine derartige Verschiedenheit bemerkt zu haben, dass er für diesen Vogel sogar eine neue Gattung creirte und ihn Cichloselys fuscatus benannte.

Von besonderem Interesse dürfte vielleicht für viele Ornithologen die Wahrnehmung sein, das schon seit mehreren Jahren das schwedische Blaukehlchen, Erithacus suecica, in die Gegend von Löwen bis nach Lüttich kommt, um dort zu nisten, und dass alle ebenso reine rostfarbige Kehlflecke haben, wie diejenigen, welche in Schweden gefangen wurden. Mithin ist also dieser Brustfleck nicht Ursache des Klima's. - Auch hält sich diese schwedische Art ganz rein neben $E$. cyanecula, welche auch in dortiger Gegend, aber seltener wie E. suecica vorkommt. Ich habe viele Alte und Junge gesehen und bemerkt, dass bei den Jungen dieser Fleck sich schon schwach rostfarbig zeigte. $\boldsymbol{E}$. Wolfi ist jedoch nichts anderes als $\boldsymbol{E}$. cyanecula; denn man trifft deren an, wo beim Zurïcklegen der blauen Federn die weissen sichtbar werden, auch ist diess ebenso bei $\boldsymbol{E}$. obcura der Fall, dessen Artverschiedenheit nicht haltbar ist und die auch zu $E$. cyanecula gehört.

Brüssel, im October 1859.

\section{Verzeichniss der Vögel des Thales Greyerz im Canton Freiburg in der Schweiz.}

Von Leon Olph-Galliard, aus Lyon.

Nach brieflichen Mittheilungen, mit Anmerkungen von

Dr. Ludwig Brelim.

Herr Olph-Galliard schreibt am 5. December 1859 aus Lyon: „Seit langer Zeit hatte ich den Entschluss gefasst, Nachrichten über die ornithologische Fauna des Thales Gre yerz zu sammeln. Ich habe geglaubt, dass eine kleine Arbeit über die Vögel dieser schönen Gegend nicht ermangeln würde, ein ganz besonderes Interesse darzubieten und das um so mehr aus dem Grunde, weil es durch seine ächte Alpenlage (par sa position toute alpestre) in den Jahrbüchern der Ornithologie berühmt zu werden verdiente. Damit eine solche Arbeit dem, was man von ihr 


\section{$2 \mathrm{BHL}$ Biodiversity Heritage Library}

1860. "Allgemeine Bemerkungen und über einige Vögel Belgiens." Journal fu

r Ornithologie 8, 224-228. https://doi.org/10.1007/bf02015746.

View This Item Online: https://www.biodiversitylibrary.org/item/101709

DOI: https://doi.org/10.1007/bf02015746

Permalink: https://www.biodiversitylibrary.org/partpdf/141558

\section{Holding Institution}

Smithsonian Libraries

\section{Sponsored by}

Biodiversity Heritage Library

\section{Copyright \& Reuse}

Copyright Status: Public domain. The BHL considers that this work is no longer under copyright protection.

This document was created from content at the Biodiversity Heritage Library, the world's largest open access digital library for biodiversity literature and archives. Visit BHL at https://www.biodiversitylibrary.org. 